\title{
Flavour physics and extra-dimensions
}

\author{
Abhishek M. Iyer ${ }^{1, *}$ \\ ${ }^{1}$ INFN-Sezione di Napoli, Via Cintia, 80126 Napoli, Italia
}

\begin{abstract}
Randall-Sundrum (RS) model of warped extra-dimensions were originally proposed to explain the Planck-weak scale hierarchy. It was soon realised that modifications of the original setup, by introducing the fields in the bulk, has several interesting features. In particular it imbues a rich flavour structure to the fermionic sector thereby offering an understanding of the Yukawa hierarchy problem. This construction is also useful in explaining the recently observed deviations in the decay of the $B$ mesons. We consider two scenarios to this effect : A) Right handed muon fields coupled more to NP that the corresponding muon doublets (unorthodox case). Non-universality exists in the right handed sector. B) Standard scenario with anomalies explained primarily by non-universal couplings to the lepton doublets. Further, we establish correlation with the parameter space consistent with the flavour anomalies in the neutral current sector and obtain predictions for rare $K$ - decay which are likely to be another candle for NP with increased precision. The prediction for rare $K$ decays are different according to the scenario, thereby serving as a useful discriminatory tool. We also discuss the large flavour violation in the lepton sector and present an example with the implementation of bulk leptonic MFV which is essential to realize the model with low KK scales. Further we consider a radical solution, called GUT RS models, where the RS geometry can work as theory of flavour in the absence of flavour symmetries. In this case the low energy brane corresponds to the GUT scale as a result of which RS is no longer solution to the gauge hierarchy problem. The Kaluza Klein (KK) modes in this setup are naturally heavy due to which the low energy constraints can be easily avoided. We use this framework to discuss the supersymmetric version of the RS model and provide means to test this scenario by considering rare lepton decays like $\tau \rightarrow \mu \gamma$.
\end{abstract}

\section{Introduction}

Flavour physics offers an indirect probe towards the possible existence of new physics (NP) effects which may be characterized by a flavour structure different from that of the SM. Process like $\mu \rightarrow e \gamma, \tau \rightarrow \mu \gamma$ in leptonic sector and rare $K$ decays in the hadronic sector are characterised by small contributions in the SM but relatively weaker experimental bounds. More recently, the anomalous decays of the $B$ mesons has generated a lot of interest. Specifically the measurement of $\mathcal{B}\left(B^{+} \rightarrow K^{+} \mu^{+} \mu^{-}\right)$and $\mathcal{B}\left(B^{+} \rightarrow K^{+} e^{+} e^{-}\right)$was quoted in form of the following ratio [1]

$$
\begin{aligned}
R_{K} & =\left.\frac{\mathcal{B}\left(B^{+} \rightarrow K^{+} \mu^{+} \mu^{-}\right)}{\mathcal{B}\left(B^{+} \rightarrow K^{+} e^{+} e^{-}\right)}\right|_{q^{2}=1-6 G e V^{2}} \\
& =0.745_{-0.074}^{+0.090}(\text { stat }) \pm 0.036(\text { syst })
\end{aligned}
$$

while the SM expectation is $R_{K}^{S M}=1.003$ [2]. This implies $\mathrm{a} \sim 2.6 \sigma$ deviation as a possible evidence of lepton nonuniversality. This observation was further validated by the

*e-mail: iyera@na.infn.it following measurement

$$
\begin{aligned}
R_{K^{*}} & =\frac{\mathcal{B}\left(B^{0} \rightarrow K^{* 0} \mu^{+} \mu^{-}\right)}{\mathcal{B}\left(B^{0} \rightarrow K^{* 0} e^{+} e^{-}\right)} \\
& =0.660_{-0.070}^{+0.110}(\text { stat }) \pm 0.024(\text { syst }), \quad \text { low } q^{2} \\
& \left.\left.=0.685_{-0.069}^{+0.113}(\text { stat }) \pm 0.047 \text { (syst }\right), \text { mid } q^{2} 2\right)
\end{aligned}
$$

where low $q^{2}$ corresponds to $0.045 \leq q^{2} \leq 1.1 \mathrm{GeV}^{2}$ while mid $q^{2}$ corresponds to $1.1 \leq q^{2} \leq 6.0 \quad \mathrm{GeV}^{2}$ The $\mathrm{SM}$ values for the corresponding $q^{2}$ bins are: $R_{K^{*}}^{S M} \simeq 0.93$ for low $q^{2}$ while $R_{K^{*}}^{S M}=1$ elsewhere. This corresponds to a $2.4 \sigma$ deviation for low $q^{2}$ and $\sim 2.5 \sigma$ for medium $q^{2}$. The deviations from the SM values can parametrized by additional contributions to the Wilson coefficients $C_{i}$ of the following effective operators: [3]:

$$
\mathcal{L} \supset \frac{G_{F} \alpha}{\sqrt{2} \pi} \sum_{i} C_{i} O_{i}
$$

where $C_{i}=C_{i}^{S M}+\Delta C_{i}$.

$$
\begin{array}{rlrl}
O_{9} & =\left(\bar{s}_{L} \gamma^{\mu} b_{L}\right)\left(\bar{l} \gamma_{\mu} l\right) & & O_{9^{\prime}}=\left(\bar{s}_{R} \gamma^{\mu} b_{R}\right)\left(\bar{l}_{\mu} l\right) \\
O_{10} & =\left(\bar{s}_{L} \gamma^{\mu} b_{L}\right)\left(\bar{l} \gamma_{\mu} \gamma^{5} l\right) & O_{10^{\prime}}=\left(\bar{s}_{R} \gamma^{\mu} b_{R}\right)\left(\bar{l} \gamma_{\mu} \gamma^{5} \overline{\mathbf{g}}\right)
\end{array}
$$

Here $\Delta C_{i}$ determines the NP contributions to the Wilson coefficients and $l$ denotes a lepton. In the following we will consider a scenario where NP effects exits in both the muon and the electron sector. 
In this talk we discuss the explanation of these anomalies in a model with a single warped extra-dimension with the following line element [4]:

$$
d s^{2}=e^{-2 A(y)} \eta_{\mu \nu} d x^{\mu} d x^{\nu}-d y^{2}
$$

where $A(y)=k|y|, k \sim \frac{M_{P l}}{4 \pi}$ and $0 \leq y \leq \pi R$. The coordinates $y=0, \pi R$ correspond to the location of the $(U V, I R)$ brane respectively. A generalization of this framework setup with a bulk custodial symmetry [5] in considered to evade constraints from $Z \rightarrow b \bar{b}$. In addition to the KK states of the SM $W, Z$, the model is characterized by additional 'custodial' heavy gauge bosons thereby leading to a distinct phenomenology, in the flavour sector in particular. A comprehensive analysis of different flavour transitions in this setup was considered in [6-9]. We explore the parameter space admitted by the current anomalies and offer predictions for the $K \rightarrow \pi v v$ decays, in the $s \rightarrow d$ sector [10]. Fits with two scenarios are demonstrated:

Scenario A. Right handed leptons are more composite (closer to the IR brane) than the left handed leptons. This is particularly true for the muon and tau. The left handed lepton couple universally to the NP. Non-universality in this case exists in the right-sector. ,

Scenario B. Left handed leptons are more composite than the right handed leptons.

For Scenario $\mathbf{A}$, the NP contribution to $\Delta C_{9,10}^{\mu}$ is dominant with a smaller contribution to $\Delta C_{9,10}^{e}$, thereby resulting a 4- $D$ fit along the lines of [11]. The primed operators do not contribute as we assume universality in the bulk wavefunctions of the right handed quarks. Both these scenarios are characterized by different predictions for the the $K$ decays thereby making it a useful discriminant.

The note is organized as follows: In Section 2 we compute the fits for the anomalies in $b \rightarrow$ sll processes for two different 1-D hypotheses. In Section 3 we consider the rare kaon decays and demonstrate how it can be utilised to possibly distinguish between the two scenarios used to fit the $b \rightarrow$ sll anomalies. In Section 4 we give an explicit example with MFV implemented in the lepton sector, in particular for the first scenario. in Section 5 we discuss a radical solution with the implementation of RS model at the GUT as a means to avoid the introduction of additional flavour symmetries and we finally conclude.

\section{$2 b \rightarrow$ sll processes : $B$ anomalies}

In bulk custodial models in RS, neutral currents at tree

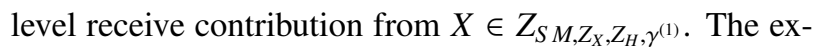
pression for the coupling of the SM fermions to these NP states can be written as:

$$
\begin{array}{r}
\mathcal{L}_{N P} \subset X_{\mu}\left[\alpha_{L}^{b s}(X)\left(\bar{s}_{L} \gamma^{\mu} b_{L}\right)+\alpha_{R}^{b s}(X)\left(\bar{s}_{R} \gamma^{\mu} b_{R}\right)+\right. \\
\left.\bar{\mu}\left(\alpha_{V}^{l}(X) \gamma^{\mu}-\alpha_{A}^{l}(X) \gamma^{\mu} \gamma^{5}\right) \mu\right]
\end{array}
$$

where $\alpha_{V, A}^{l}(X)=\frac{\alpha_{L}^{l}(X) \pm \alpha_{R}^{l}(X)}{2}$. $\alpha$ gives the coupling in the zero mode fermions to the gauge bosons in the mass basis of the latter. Using these expressions, the Wilson co- efficients for each gauge field $X$ are written as:

$$
\begin{aligned}
\Delta C_{9} & =-\frac{\sqrt{2} \pi}{M_{X}^{2} G_{F} \alpha} \alpha_{L}^{b s}(X) \alpha_{V}^{l}(X), \\
\Delta C_{10} & =\frac{\sqrt{2} \pi}{M_{X}^{2} G_{F} \alpha} \alpha_{L}^{b s}(X) \alpha_{A}^{l}(X),
\end{aligned}
$$

For the primed operators the expressions are similar with $L \leftrightarrow R$. In the above we assumed that the up-sector quark are in the mass-diagonal basis and down quark rotation matrices are $D_{L, R} \sim V_{C K M}$. We now discuss the following two possibilities for the fits to the data:

1) Scenario A: This scenario is characterized by the relatively larger contribution of the lepton singlets to the NP than the doublets. The doublets are chosen to have to have universal bulk wavefunction with $c>0.5$. This choice is particularly helpful in obtaining an anarchic neutrino mixing matrix which is roughly given as $U_{P M N S}^{i j} \sim$ $\frac{f\left(c_{L}^{i}\right)}{f\left(c_{L}^{j}\right)}$

The contributions to $\Delta C_{9,10}^{\prime}$ are made consistent with zero by assuming that the right handed down quarks couple similarly to the NP. Numerically this implies $c_{d_{R}, s_{R}, b_{R}}>$ 0.55 . The ranges chosen for $c$ parameter scan are chosen to be: $c_{Q_{3}} \in[0,0.5], c_{\mu_{L}}=c_{L} \in[0.51,0.6]$ and $c_{\mu_{R}} \in[0.45,0.55]$. Further by choosing the $c$ parameters for the first two quark generations $c_{Q_{1,2}}>0.55$ ensures a universality of their coupling to the NP states. Fig. 1 gives the results of the scan: The top plot gives the correlation between $\Delta C_{9}^{\mu}-\Delta C_{9}^{e}$ while the bottom gives the correlation between $\Delta C_{10}^{\mu}-\Delta C_{10}^{e}$. The 2- $\sigma$ regions for a $4 \mathrm{D}$ fit to the data is [11]

$$
\begin{array}{r}
C_{9}^{\mu} \in[-0.33,0.06] \quad C_{9}^{e} \in[-2.23,0.74] \\
C_{10}^{\mu} \in[-0.29,0.14] \quad C_{10}^{e} \in[-2.60,0.60]
\end{array}
$$

The non-negligible values of the $\Delta C^{e}$ is due to left doublets having $c \sim 0.5$ thereby resulting in a mildly larger coupling to the NP states than would be expected of states having $c \geq 0.55$. For these choices of $c$ parameters corresponding to the values in Fig.1, fitting the muon mass requires choosing the $O(1)$ Yukawa $\sim 0.03$. Though slightly fine tuned with regards to the fit to the muon mass, this scenario is more favorable with regards to an anarchic neutrino mixing matrix but also in suppressing FCNC in the lepton sector through the implementation of 5D MFV.

2) Scenario B: In this case the non-universality is now transferred to the lepton doublets while the singlets are closer to the UV brane and their coupling to the NP is universal. Further, without loss of generality we assume that the $\tau$ doublets are closer to the IR brane than the $\mu$ doublets $\left(c_{\tau_{L}}<c_{\mu_{L}}\right)$. All the lepton singlets and the electron doublet satisfy $c>0.55$. This results in $\Delta C_{9,10}^{e}$ much smaller than $\Delta C_{9,10}^{\mu}$, with its magnitude being at most $\sim 0.2$. For most of the region, it effectively reduces this to a 2-D fit where the value of $C_{9,10}^{e}$ is an order of magnitude less,

Top left plot of Fig.2 gives the correlation in the $\Delta C_{9}^{\mu}-\Delta C_{10}^{\mu}$ plane which correspond to points which sat- 

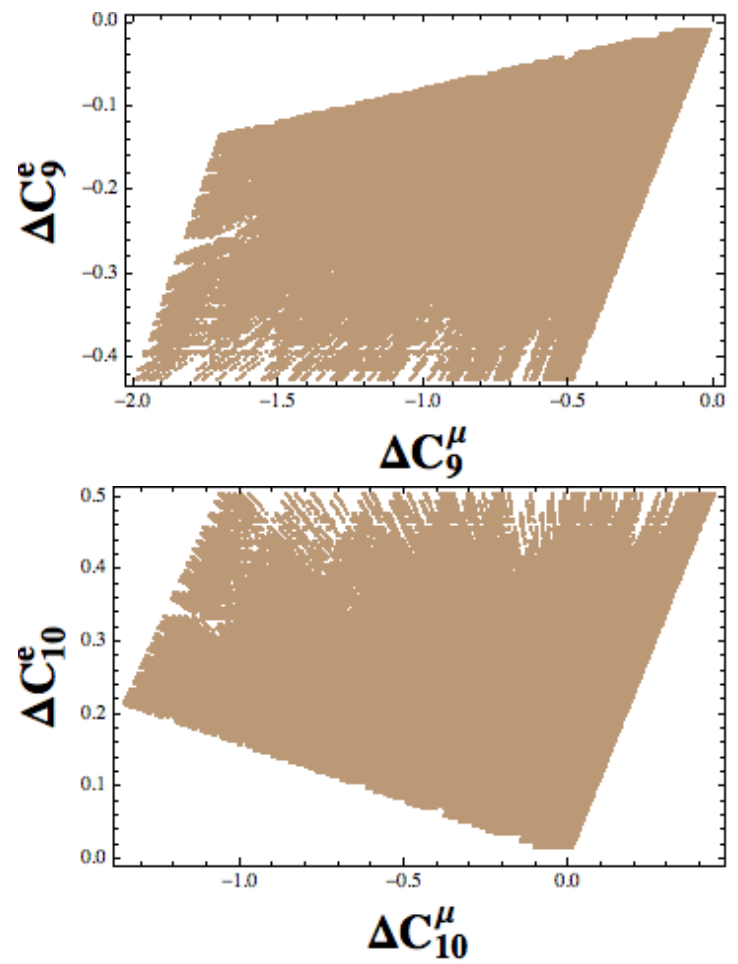

Figure 1. Scenario A: Plot show the correlation in the $C_{9}$ and $C_{10}$ parameter plane for both the electron and the muon. We use $M_{K K}=3 \mathrm{TeV}$

isfy $0.36<\left|\Delta C_{9,10}^{\mu}\right|<0.87$. There also exist solutions for which $\Delta C_{9}^{\mu}=-\Delta C_{10}^{\mu}$ further reducing it to a 1-D fit as discussed in $[18,19]$. The only two relevant parameters for the fits to the $\mathrm{B}$-anomalies are $c_{Q_{3}}-c_{\mu_{L}}$ and the correlation is shown in right plot of Fig. 2.

\section{Kaon decays}

In this section we use rare $K$ decays to differentiate between the two scenarios considered earlier. $K^{+} \rightarrow \pi^{+} v v$ and $K_{L} \rightarrow \pi^{0} v v$ are likely to constitute the next probe towards the possible existence of NP. They correspond to $s \rightarrow d v v$ form of transitions and are likely to be correlated to $b \rightarrow$ sll transitions in most NP scenarios. The SM value for the $K^{+} \rightarrow \pi^{+} v \bar{v}$ and $K_{L} \rightarrow \pi^{0} v \bar{v}$ is $[12,13,21]$ :

$$
\begin{aligned}
\mathcal{B}\left(K^{+} \rightarrow \pi^{+} v \bar{v}\right) & =8.3 \pm 0.3 \pm 0.3 \times 10^{-11} \\
\mathcal{B}\left(K_{L} \rightarrow \pi^{0} v \bar{v}\right) & =2.9 \pm 0.2 \pm 0.0 \times 10^{-11}
\end{aligned}
$$

where the first error is due to the uncertainty in the parameters of $V_{C K M}$ mixing matrix and the second one is due to the remaining theoretical uncertainties. The current experimental bound is [22]

$$
\begin{aligned}
\mathcal{B}\left(K^{+} \rightarrow \pi^{+} v \bar{v}\right) & =17.3_{-10.5}^{+11.5} \times 10^{-11} \\
\mathcal{B}\left(K_{L} \rightarrow \pi^{0} v \bar{v}\right) & \leq 2.6 \times 10^{-8} \quad(90 \% \text { C.L. })
\end{aligned}
$$

These measurements are likely to be significantly improved in the future. The NA62 experiment at CERN $[14,15]$ is pursuing to reach a precision of $15 \%$ compared to the $\mathrm{SM}$ in 2018 . while $5 \%$ accuracy will be
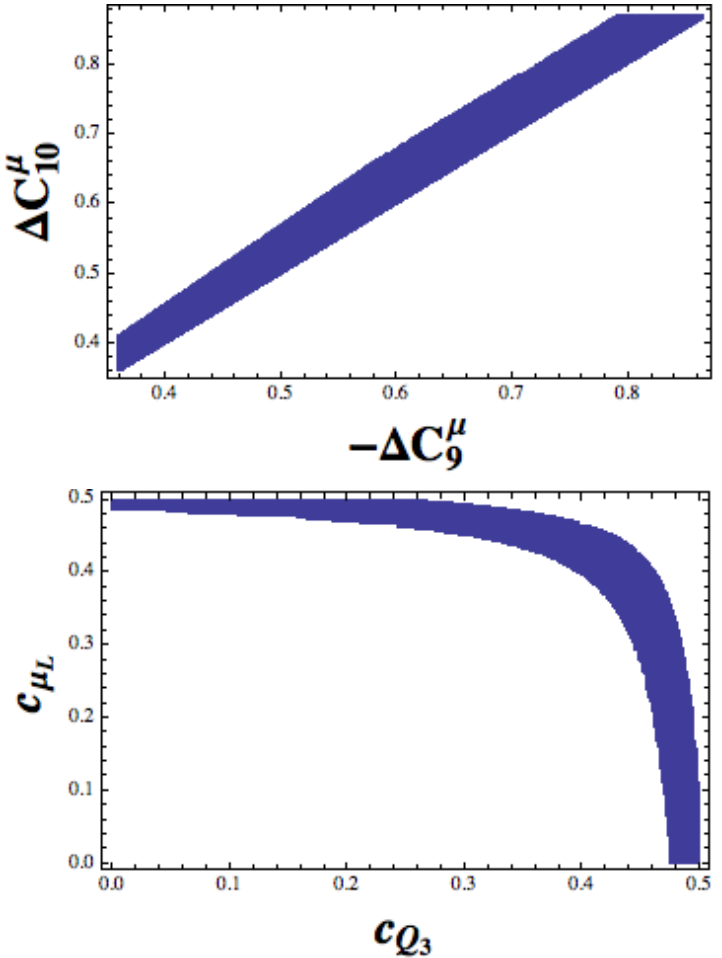

Figure 2. Scenario $\mathbf{B}$ : Top plot gives the distribution for $\Delta C_{9}$ and $\Delta C_{10}$. The corresponding $c$ parameters ranges are given in the bottom plot.

achieved with more time. With regards to the $K_{L} \rightarrow$ $\pi^{0} v \bar{v}$, the KOTO experiment at J-PARC aims at measuring $\mathcal{B}\left(K_{L} \rightarrow \pi^{0} v \bar{v}\right)$ around the SM sensitivity in the first instance [16, 17]. Moreover, the KOTO-step2 experiment will aim at 100 events for the SM branching ratio. This implies a precision of $10 \%$ of this measurement.

These processes can be described by the following effective Lagrangian parameterizing for $s \rightarrow d v v$ transitions:

$$
\mathcal{L}=\frac{4 G_{F} \alpha}{2 \sqrt{2} \pi} V_{t s}^{*} V_{t d} C_{d s, l}\left(\bar{s}_{L} \gamma_{\mu} d_{L}\right)\left(\bar{v}_{l} \gamma^{\mu} v_{l}\right)
$$

The Wilson co-efficient $C_{d s, l}$ in the SM is given as:

$$
C_{d s, l}^{S M}=-\frac{1}{s_{\theta_{w}}^{2}}\left(X_{t}+\frac{V_{c s}^{*} V_{c d}}{V_{t s}^{*} V_{t d}} X_{c}^{l}\right)
$$

where $X_{t}$ and $X_{c}^{l}$ are the loop functions for the top and charm contribution respectively and given as: $X_{t}=1.481 \pm$ 0.009 and $\frac{1}{3} \sum_{l} \frac{X_{c}^{l}}{\lambda^{4}}=0.365 \pm 0.012$ [20] the branching ratio for $K^{+} \rightarrow \pi^{+} v v$ is given as:

$$
\begin{aligned}
\mathcal{B}\left(K^{+} \rightarrow \pi^{+} v v\right) & =\frac{\kappa_{+}\left(1+\Delta_{e m}\right)}{3} \sum_{l=e, \mu, \tau} \mid \frac{V_{t s}^{*} V_{t d}}{\lambda^{5}} X_{t} \\
& +\left.\frac{V_{c s}^{*} V_{c d}}{\lambda}\left(\frac{X_{c}^{l}}{\lambda^{4}}+\delta P_{c}^{l}\right)\right|^{2} \\
\mathcal{B}\left(K_{L} \rightarrow \pi^{0} v v\right) & =\frac{\kappa_{L}}{3} \sum_{l=e, \mu, \tau}\left(\frac{\operatorname{Im}\left(V_{t s}^{*} V_{t d}\right)}{\lambda^{5}} X_{t}\right)^{2}
\end{aligned}
$$


where $\kappa_{L}=2.231 \pm 0.013 \times 10^{-10}(\lambda / 0.225), \kappa_{+}=5.173 \pm$ $0.025 \times 10^{-11}(\lambda / 0.225), \Delta_{e m}=-0.003$ [23] and $\delta P_{c, u}^{l}=$ $0.04 \pm 0.02$ [24]. The individual values of $X^{l}$ were obtained from Table 1 of [25]: $X^{e, \mu}=11.18 \times 10^{-4}, X^{\tau}=7.63 \times 10^{-4}$.

We now consider the NP contributions to the process $s \rightarrow d l l$ given in Eq. 12. In the bulk custodial model under consideration, the effective lagrangian for the process is given as

$$
\mathcal{L}_{s \rightarrow d v v} \equiv\left[\alpha_{L}^{s d}\left(\bar{s}_{L} \gamma^{\mu} d_{L}\right)+\alpha_{R}^{s d}\left(\bar{s}_{R} \gamma^{\mu} d_{R}\right)\right]\left(v_{L} \bar{\gamma}_{\mu} v\right) \alpha_{L}^{l}
$$

In general this includes both the left handed and the right handed current in the quark sector signaling a possible deviation from the $(V-A)(V-A)$ structure given in Eq. 12 . This aspect was explored in great detail in [7]. We discuss this process in the context of the two scenarios discussed in Section 2. It is worth stressing at this point that the $s \rightarrow d v v$ transitions only depend on the left handed $c_{L}$ parameters for the leptons, while both $c_{Q_{3}}$ and $c_{b_{R}}$ play a role. However, since we assumed only the third generation doublets to have $c_{Q_{3}}<0.5$, there are no tree-level FCNC in the right handed sector. The contribution can be quantified by making the following change to the $X_{t}$ in Eq. 13:

$$
X_{t} \rightarrow X_{t}+\sum_{X=Z_{X, H} \gamma^{(1)}} \frac{\sqrt{2} 2 \pi}{4 G_{f} \alpha} \frac{\alpha^{s d}(X) \alpha_{L}^{l}(X)}{M_{K K}^{2}}
$$

where as earlier the contribution due to $Z_{X}$ is suppressed. The SM limit is computed in the limit $M_{K K} \rightarrow \infty$.

We consider the following ratio for both the decays $\mathcal{B}_{\text {total }}^{i} / \mathcal{B}_{S M}^{i}$ for $i=K_{L}, K^{+}$and evaluate it for the two scenarios discussed earlier:

1) Scenario A: This case is characterized by the universality in the left handed lepton sector. Since neutrinos in the final state are left handed, only $c_{L}$ (parameter for the lepton doublets) will play a role its computation. To stress the fact that $B$ anomalies are explained purely due to non-universality in the right handed sector for leptons we choose : $c_{\mu_{R}} \sim 0.48$ for the muon singlet while $c_{L} \sim 0.51$ for all three generations. Fig. 3 gives plot of $\mathcal{B}_{\text {total }}^{i} / \mathcal{B}_{S M}^{i}$ computed as a function of $c_{Q_{3}}$ and evaluated for $c_{L}=0.51$. This corresponds to the parameter space of the hypothesis under consideration. It can be seen that for both the decays, the ratio is very close to the SM prediction thereby predicting no net enhancement. In principle one can choose to reduce $c_{Q_{3}}$ lesser than 0 at the cost of increasing its compositeness and possible tension with $Z \rightarrow b \bar{b}$ constraints.

2) Scenario B: This case is characterized by nonuniversality in the left handed lepton sector while the NP coupling to the right handed singlets are universal. Fig. 4 gives the ratio $\mathcal{B}_{\text {total }}^{i} / \mathcal{B}_{S M}^{i}$ for both the kaon decays as a function of $c_{\mu_{L}}$ and $c_{Q_{3}}$. We note that for this scenario where $c_{\mu_{L}}<0.5$, the region consistent with the $b \rightarrow$ sll leads to enhancement of $\sim 1.2-1.6$, depending on the value of $c_{\mu_{L}}$ and $c_{Q_{3}}$. This is an useful example where a more accurate measurement of certain process may help in narrowing down the NP parameter space.
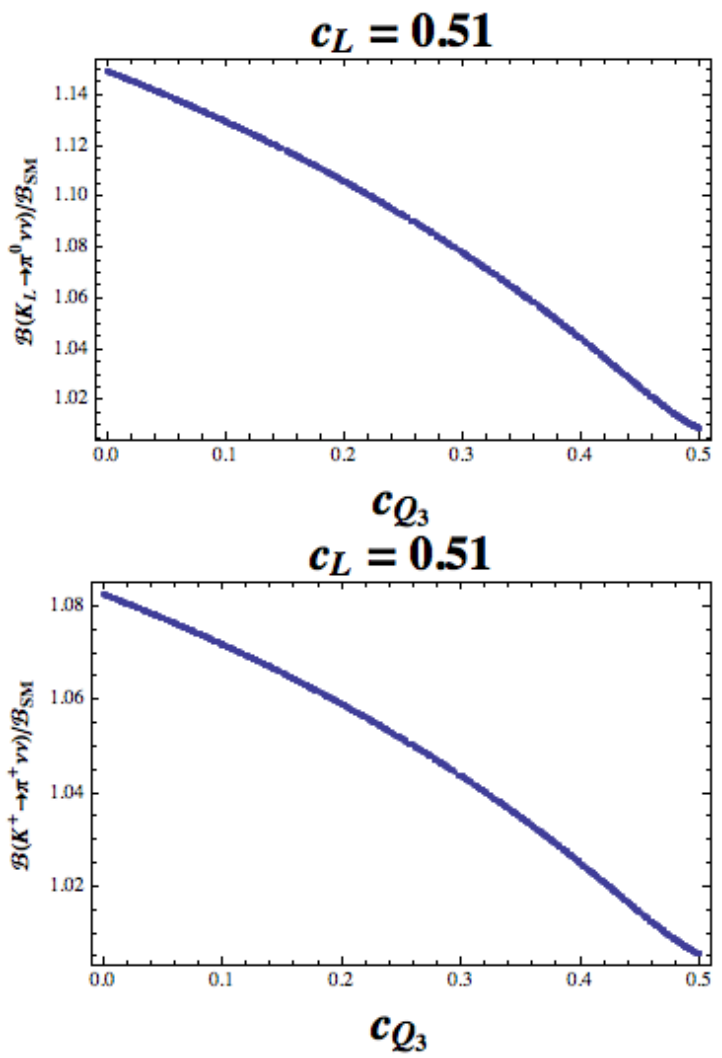

Figure 3. Scenario A: Plots depicting the excess over the SM expectation for the $K$ decays modes. The $c$ parameters for the doublets is universal and chosen to be $c_{L}=0.51$.

\begin{tabular}{|c|c|}
\hline Process & Experimental(Upper Bound) \\
\hline $\mathcal{B}(\mu \rightarrow$ e $)$ & $4.2 \times 10^{-13}[27]$ \\
$\mathcal{B}(\mu \rightarrow$ eee $)$ & $1.1 \times 10^{-12}[28]$ \\
$\mathcal{B}(\mu-e)$ Conv $(\mathrm{Ti})$ & $6.1 \times 10^{-13}[29]$ \\
\hline
\end{tabular}

Table 1. Experimental upper bound for the branching fraction of leptonic flavour observables in the 1-2 sector.

\section{Leptonic MFV}

The localization of the fermions at different points in the bulk leads to their non-universal coupling to the gauge KK states, These typically give rise to additional contributions to different to FCNC processes at tree level. Thus this is an example of a scenario where lepton non-universality leads to flavour violation. In minimal setup and for $\mathrm{KK}$ scales within the reach of LHC, these contributions can be particularly large in the lepton sector. This is mainly due to the strong upper bounds on processes in the 1-2 sector $\mu \rightarrow e \gamma, \mu \rightarrow e e e, \mu-e$ conversion [26]. The current experimental upper bounds on these processes are given in Table 1. The large contributions can be attributed to the misalignment between the Yukawa coupling matrix and the bulk mass parameters which determine the nature of the fermionic profiles in the bulk. A complete model explaining anomalies in $B$ sector with a relatively low NP scale must also satisfy constraints given in Table 1 . 

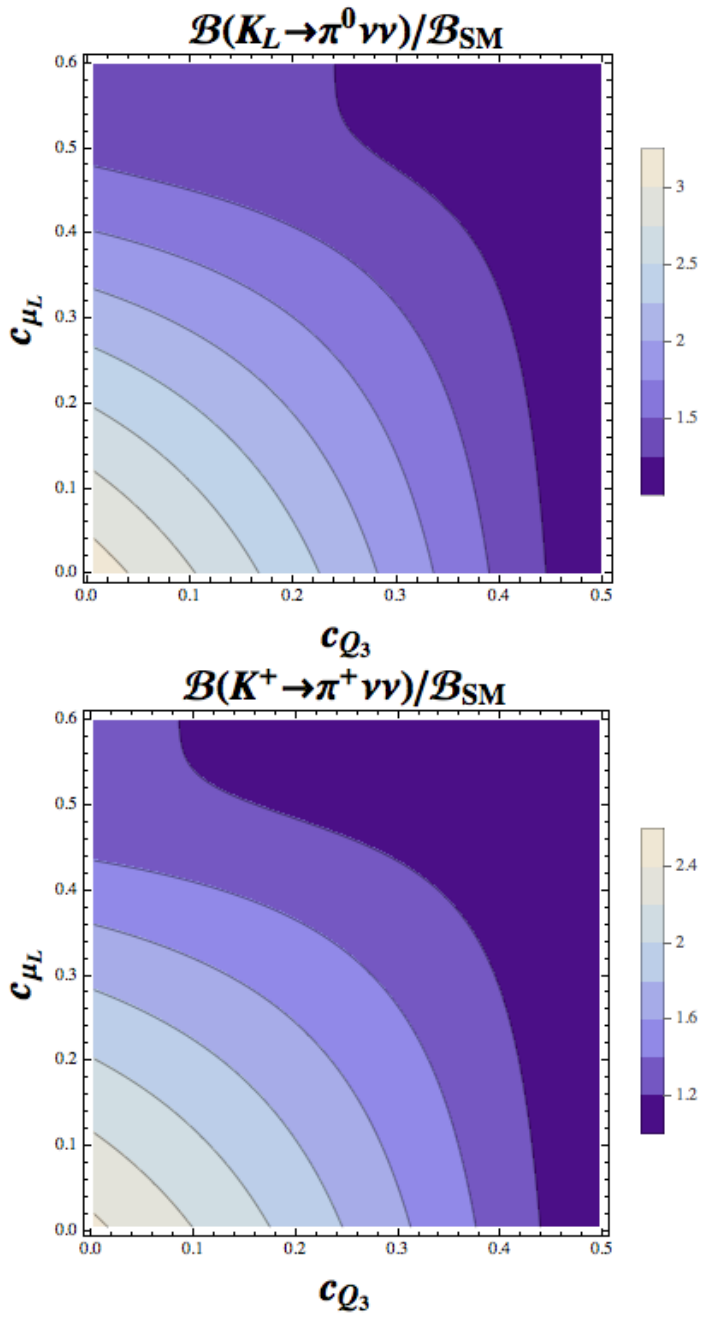

Figure 4. Scenario B: Plots depicting the excess over the SM expectation for the $K$ decays modes. $c_{\tau_{L}}=0.4$ and $c_{e_{L}}=0.6$ are fixed for the computation while $c_{\mu_{L}}$ is varied.

One possibility to alleviate these constraints is to consider the implementation of MFV in 5D [30]. The large contributions to FCNC are primarily due to the misalignment between the flavour violating combination $Y Y^{\dagger}$ and the mass-squared matrix $m^{2} \sim\left(F(c) Y Y^{\dagger} F(c)\right)$. Here $F(c)$ is the diagonal matrix of bulk profiles given as $F(c)=$ $\operatorname{Diag}\left(f\left(c_{e}\right), f\left(c_{\mu}\right), f\left(c_{\tau}\right)\right)$. Since $Y$ and $F(c)$ do not commute in general, diagonalization of $m^{2}$ does not necessarily imply diagonalization of $Y Y^{\dagger}$. This misalignment can be reduced by making the specific choice where the $5 \mathrm{D}$ Yukawa parameters are written in terms of the bulk mass parameters as follows:

$$
\begin{aligned}
c_{L} & =a_{L} I+b_{L} Y_{E} Y_{E}^{\dagger}+d_{L} Y_{N} Y_{N}^{\dagger} \\
c_{E} & =a_{E} I+b_{E} Y_{E}^{\dagger} Y_{E} \\
c_{N} & =a_{N}+b_{N} Y_{N}^{\dagger} Y_{N}
\end{aligned}
$$

where $a_{i}, b_{i} \in \mathfrak{R}$. We also assume the presence of a bulk flavour symmetry group in the leptonic sector as $S U(3)_{L} \times S U(3)_{E} \times S U(3)_{N}$. Using the flavour symmetry we can work in a basis in which $Y_{E}$ is diagonal. Thus flavour violations are embedded in $Y_{N}$ which transforms under the flavour group as $Y_{N} \rightarrow V_{5} Y_{N}$ where $V_{5}$ is the anarchic mixing matrix. Without loss of generality we can choose $V_{5}=V_{P M N S}$. To enable the explanation of the $B$ anomalies, we assume that the corresponding bulk flavour symmetry in the hadronic sector is broken with the corresponding $c$ parameters not satisfying to similar relations like Eq. 17 in the hadronic sector. We now discuss its implementation for the two scenarios discussed in Section 2. a) Scenario A: In this scenario the lepton doublets are localized closer to the UV brane and are assumed to have universal coupling to NP. The $\mu_{R}$ is relatively closer to the IR brane We assume $c_{L} \simeq 0.51$ for all three generations. The $c$ values for the charged lepton singlets are chosen as $c_{E}=\{0.4,0.48,0.764\}$ and corresponding $O(1)$ Yukawa couplings are $Y_{E}=\{0.77,0.03,0.32\}$. The $c_{E}$ are written in terms of $Y_{E}$ by choosing $a_{E}=0.49, b_{E}=-0.84 \mathrm{in} \mathrm{Eq.}$ 17. For $c_{L}$ we choose $a_{L} \gg b_{L}, d_{L}$ to preserve the universality for the left handed doublets.

To fit the neutrino mass date we make choices for $c_{N}$ and $Y_{N}$ as: $c_{N}=\{1.17,1.17,1.21\}$ and $\operatorname{Diag}\left(Y_{N}\right) \equiv$ $\{0.1,0.1,0.134\}$ leading to the following fit for the neutrino oscillation data:

$$
\Delta m_{\text {sol }}^{2}=7.7 \times 10^{-5} e V^{2} \quad \Delta m_{\text {atm }}^{2}=1.98 \times 10^{-3} e V^{2}
$$

which corresponds to an inverted hierarchy spectrum.

Corresponding to these choices, the $\mathcal{B}(\mu \rightarrow e \gamma)$ is given as [31]:

$$
\mathcal{B}(\mu \rightarrow e \gamma)=4 \times 10^{-8} \times\left(Y_{N} Y_{N}^{\dagger}\right)_{12}^{2} \frac{3 T e V}{M_{K K}}
$$

where

$$
Y_{N}=\left[\begin{array}{ccc}
0.0823679 & 0.0548227 & 0.0194184 \\
-0.0477093 & 0.0531867 & 0.0937521 \\
0.0306488 & -0.0645418 & 0.0937521
\end{array}\right]
$$

leading to $\mathcal{B}(\mu \rightarrow e \gamma) \sim 2.5 \times 10^{-14}$. Similarly the other $1-2$ transitions are also within the experimental bounds quoted in Table 1 for $M_{K K}=3 \mathrm{TeV}$.

b) Scenario B: In the earlier case we saw that the universality of the lepton doublet parameters $c_{L}$ was essential to implement the MFV ansatz and obtain satisfactory fits to the neutrino mases. Universality ensures that the values of the rotation matrix $U_{i j} \sim \frac{f_{L_{i}}}{f_{L_{i}}} \sim O(1)$ which roughly correspond to the elements of the PMNS rotation matrix. Since the $c_{L}$ is not universal in this scenario, fits to the neutrino data also require hierarchical choices in $c_{N}$. This makes the implementation of $c_{N}$ proportional to $Y_{N}^{\dagger} Y_{N}$ in 17 extremely challenging and difficult to achieve. This possibly requires a more complicated parameter scan and will not be discussed here.

\section{Randall Sundrum model at the GUT scale}

Given these strong constraints on the RS set up at the weak scale, one can ask the question whether RS is suitable to be a theory of flavour as well as a solution to the hierarchy 
problem simultaneously. It might be that RS as a theory of a flavour might be better suited at the GUT scale rather than at the weak scale. The Froggatt-Nielsen models are typically defined at scales closer to the Planck scale, so perhaps flavour physics might have its origins at the Planck scale. With this point of view, we will now consider RS to span between the Planck and the GUT scales. As a result the lowest lying KK scales are $O\left(M_{G U T}\right)$ and are decoupled from the low energy theory. Introducing bulk supersymmetry serves as a solution to the hierarchy problem at the weak scale. The supersymmetric case has the added advantage that it could lead to observable signatures at the weak scale and will be the focus of attention in this section [35].

In addition to the matter fields, we consider the the case with bulk Higgs doublets $\left(H_{u, d}\right)$ and a SUSY breaking spurion $X$. The Kähler terms, leading to non-negative contributions to the scalar masses are given as [32,33]

$$
\mathcal{K}=\sum_{M=Q, U, D, L, E} e^{-2 \sigma(y)} \delta\left(y-y_{0, \pi R}\right) \frac{\hat{C}_{i j}}{M_{p l}^{4}} X^{\dagger} X M^{\dagger} M
$$

Replacing $X \equiv\langle F\rangle$, leads to the non-tachyonic soft masses as

$$
\begin{aligned}
m_{i j}^{2} & =m_{3 / 2}^{2} r^{2} \hat{m}_{i j}\left(\xi_{U V}\left(c_{i}\right) \xi_{U V}\left(c_{j}\right) \xi_{U V}^{2}\left(c_{S}\right)\right. \\
& \left.+\frac{1}{\epsilon^{2}} \xi_{I R}\left(c_{i}\right) \xi_{I R}\left(c_{j}\right) \xi_{I R}^{2}\left(c_{S}\right)\right)
\end{aligned}
$$

and where

$$
\xi_{U V}(c)=\sqrt{\frac{1-2 c}{\epsilon^{2 c-1}-1}} \quad \xi_{I R}(c)=\sqrt{\frac{1-2 c}{1-\epsilon^{2 c-1}}}
$$

and where the dimensionless parameter $r$ is defined as $r=\frac{k}{M_{5}}$ with $M_{5}$ being the fundamental 5D Planck scale. Typically $r<1$ so that the assumption of a classical gravity is valid. The gravitino mass $m_{3 / 2}=\frac{\langle F\rangle}{M_{5}}$ is the ratio of the vacuum expectation value of the F-term of the SUSY breaking field $X$ to the Planck scale. In addition to this, the theory also leads to tachyonic masses at $M_{G U T}$ and are given as [34]

$$
m_{\text {tachyonic }}^{2}\left(c_{m}, c_{s}\right)=-2 m_{3 / 2}^{2}\left(1+2 \alpha_{m s}\right)
$$

where $1+2 \alpha_{m s}=\frac{\left(1-2 c_{m}\right)\left(2-2 c_{s}\right)}{2\left(4-2 c_{m}-2 c_{s}\right)}\left(\frac{\left(1-\epsilon^{\left.3-2 c_{m}\right)}\right)\left(1-\epsilon^{3-2 c_{s}}\right)}{\epsilon^{2}\left(1-\epsilon^{\left.1-2 c_{m}\right)}\right)\left(1-\epsilon^{1-2 c_{s}}\right)}-1\right)$. Owing to the presence of these tachyonic terms, which are more prominent for the third generation quarks and less mildly for the thrid generation lepton, large values of $M_{3}$ are required so as to drive the masses to positive values by RGE effects. Further, owing to the tachynic masses of $\tilde{\tau}$, $M_{2}$ must be greater that $M_{2}$ to avoid the presence of tachyonic staus in the model. This can be seen from Fig. 5 Which predominantly prefers $M_{2}>M_{1}$. This figure was obtained using analytic expressions for one-loop RGE in the limit $Y_{\tau} \rightarrow 0$.

The flavoured masses in Eq. 22 has interesting phenomenological implications. It leads to mixing between different fermionic generations possibly leading to new contributions to FCNC. Defining the flavour violating parameter as $\delta_{i j}=\frac{\tilde{m}_{i j}^{2}}{\sqrt{\tilde{m}_{i}^{2} \tilde{m}_{j}^{2}}} \quad i \neq j$, which controls the extent

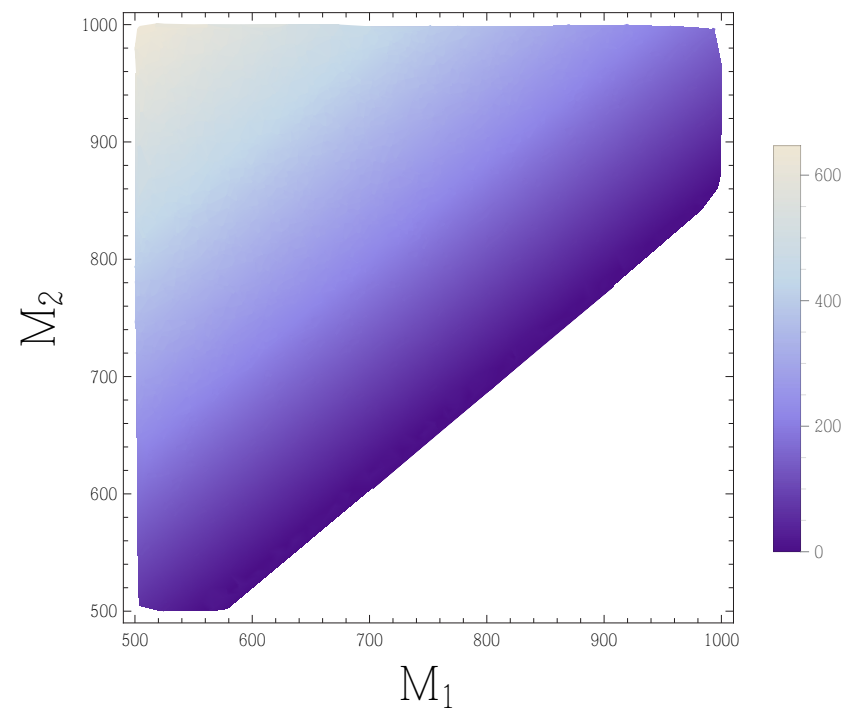

Figure 5. Lightest stau eigenstate a a function of $M_{1,2}$

of flavour violating in the theory. Fig. 6 gives the running of $\delta$ in the leptonic setor.
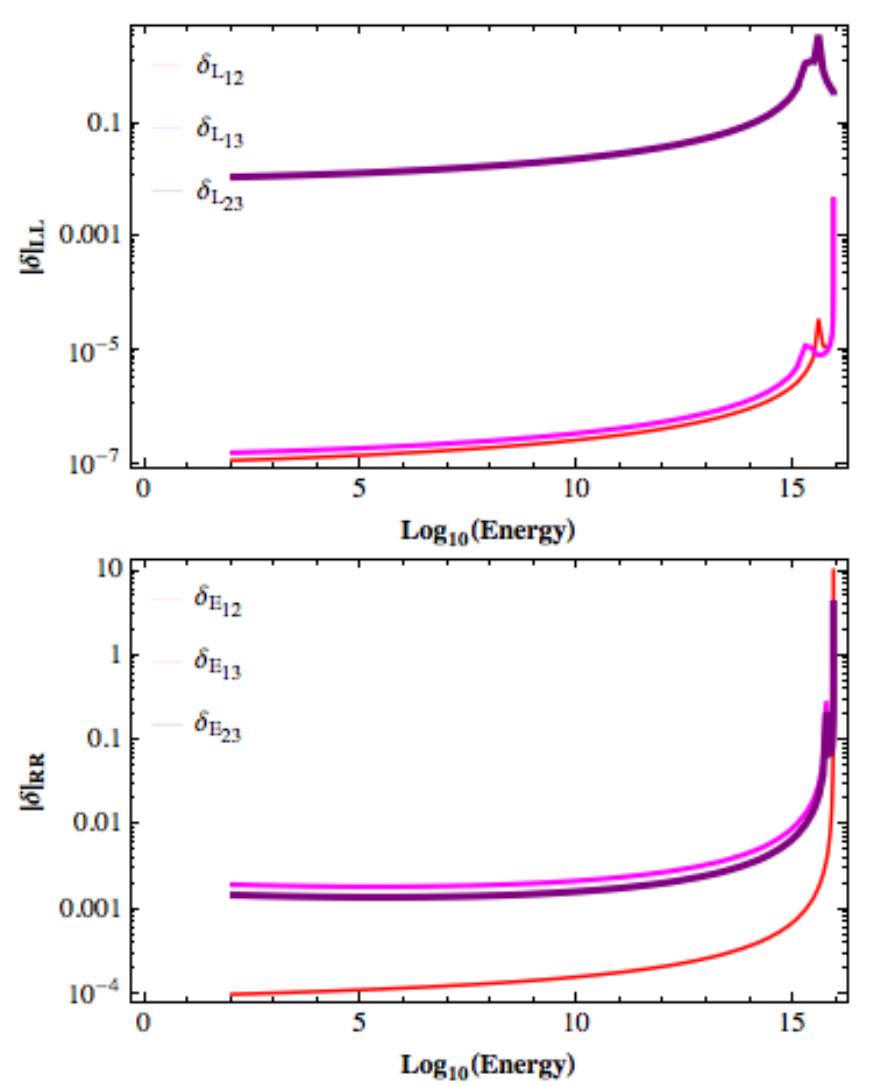

Figure 6. Running of $\delta$ for the leptonic sector.

Though $O(1)$ at the high scale, it runs down to acceptably small values leading to possible signatures of these scenarios through FCNC processes. For instance Fig. 7 gives the correlation between $\tau \rightarrow \mu \gamma, \mu \rightarrow e \gamma$ and 


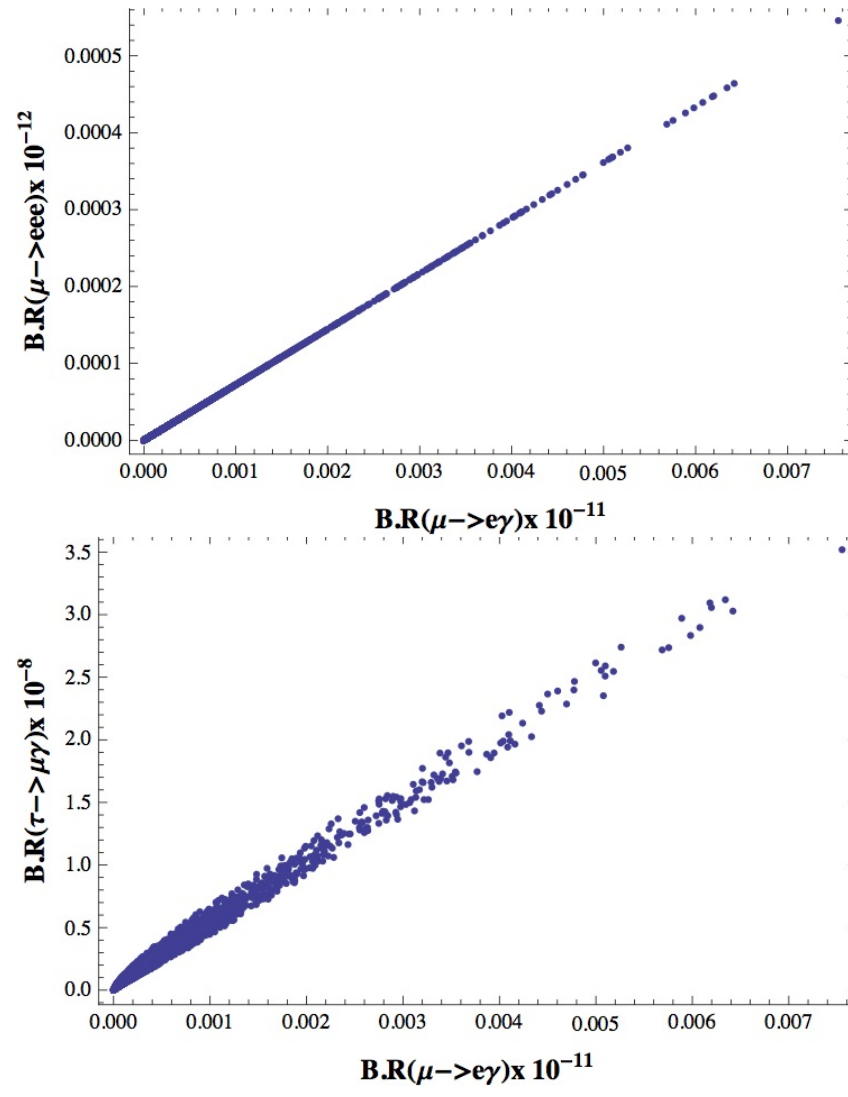

Figure 7. Correlation between $\mu \rightarrow e \gamma$ and $\mu \rightarrow e e e$ (left) and $\mu \rightarrow e \gamma$ and $\tau \rightarrow \mu \gamma$

$\mu \rightarrow$ eee. Since the squarks are decoupled from the theory, there are no observable FCNC effects in the squark sector.

\section{Conclusions}

Custodial RS models offers an interesting perspective to explore the recently observed anomlies in the decays of the $B$ mesons. We considered two scenarios which differ in the relative localization (compositeness) of the lepton doublets and singlets in the bulk. For Scenario A, characterized by universal lepton doublet wavefunction, the implementation of anarchic neutrino mixing and bulk leptonic MFV is simpler. Thereby the flavour violation in the lepton sector are under control thereby paving the way for a more complete setup. The rare $K$ decays are however consistent with the SM expectation. Finally we discuss a scenario with GUT RS where the model serves a theory of flavour while the effective low energy theory is MSSM. This has interesting implications for flavour in the lepton sector.

\section{Acknowledgements}

I would like to thank the organizers of FCCP 2017 for the opportunity to present my work in the workshop. I am also grateful to my collaborators: Giancarlo D'Ambrosio, Emilian Dudas, Sudhir Vempati for the fruitful collaborations which have lead to the interesting results presented in the workshop.

\section{References}

[1] R. Aaij et al. [LHCb Collaboration], Phys. Rev. Lett. 113, $151601 \quad$ (2014) doi:10.1103/PhysRevLett.113.151601 [arXiv:1406.6482 [hep-ex]].

[2] M. Bordone, G. Isidori and A. Pattori, Eur. Phys. J. C 76, no. 8, 440 (2016) doi:10.1140/epjc/s10052-0164274-7 [arXiv:1605.07633 [hep-ph]].

[3] G. Buchalla, A. J. Buras and M. E. Lautenbacher, Rev. Mod. Phys. 68, 1125 (1996) doi:10.1103/RevModPhys.68.1125 [hep-ph/9512380].

[4] L. Randall and R. Sundrum, Phys. Rev. Lett. 83, 3370 (1999) doi:10.1103/PhysRevLett.83.3370 [hep$\mathrm{ph} / 9905221]$.

[5] K. Agashe, A. Delgado, M. J. May and R. Sundrum, JHEP 0308, 050 (2003) doi:10.1088/11266708/2003/08/050 [hep-ph/0308036].

[6] M. Blanke, A. J. Buras, B. Duling, S. Gori and A. Weiler, JHEP 0903, 001 (2009) doi:10.1088/11266708/2009/03/001 [arXiv:0809.1073 [hep-ph]].

[7] M. Blanke, A. J. Buras, B. Duling, K. Gemmler and S. Gori, JHEP 0903, 108 (2009) doi:10.1088/11266708/2009/03/108 [arXiv:0812.3803 [hep-ph]].

[8] M. Bauer, S. Casagrande, U. Haisch and M. Neubert, JHEP 1009, 017 (2010) doi:10.1007/JHEP09(2010)017 [arXiv:0912.1625 [hep-ph]].

[9] S. Casagrande, F. Goertz, U. Haisch, M. Neubert and T. Pfoh, JHEP 1009, 014 (2010) doi:10.1007/JHEP09(2010)014 [arXiv:1005.4315 [hep-ph]].

[10] G. D'Ambrosio and A. M. Iyer, arXiv:1712.08122 [hep-ph].

[11] T. Hurth, F. Mahmoudi and S. Neshatpour, Nucl. Phys. B 909, 737 (2016) doi:10.1016/j.nuclphysb.2016.05.022 [arXiv:1603.00865 [hep-ph]].

[12] A. J. Buras, M. Gorbahn, U. Haisch and U. Nierste, JHEP 0611, 002 (2006) Erratum: [JHEP 1211, 167 (2012)] doi:10.1007/JHEP11(2012)167, 10.1088/11266708/2006/11/002 [hep-ph/0603079].

[13] A. J. Buras, D. Buttazzo, J. Girrbach-Noe and R. Knegjens, JHEP 1511, $033 \quad$ (2015) doi:10.1007/JHEP11(2015)033 [arXiv:1503.02693 [hep-ph]].

[14] A. Romano, arXiv:1411.6546 [hep-ex].

[15] F. Newson et al., arXiv:1411.0109 [hep-ex].

[16] T. K. Komatsubara, Prog. Part. Nucl. Phys. 67, 995 (2012) doi:10.1016/j.ppnp.2012.04.001 [arXiv:1203.6437 [hep-ex]].

[17] K. Shiomi [KOTO Collaboration], arXiv:1411.4250 [hep-ex].

[18] B. Capdevila, A. Crivellin, S. Descotes-Genon, J. Matias and J. Virto, arXiv:1704.05340 [hep-ph].

[19] W. Altmannshofer, P. Stangl and D. M. Straub, Phys. Rev. D 96, no. 5, 055008 (2017) doi:10.1103/PhysRevD.96.055008 [arXiv:1704.05435 [hep-ph]]. 
[20] G. Buchalla and A. J. Buras, Nucl. Phys. B 548, 309 (1999) doi:10.1016/S0550-3213(99)00149-2 [hep$\mathrm{ph} / 9901288]$.

[21] J. Brod, M. Gorbahn and E. Stamou, Phys. Rev. D 83, 034030 (2011) doi:10.1103/PhysRevD.83.034030 [arXiv:1009.0947 [hep-ph]].

[22] C. Patrignani et al. [Particle Data Group], Chin. Phys. C 40, no. 10, 100001 (2016). doi:10.1088/1674$1137 / 40 / 10 / 100001$

[23] F. Mescia and C. Smith, Phys. Rev. D 76, 034017 (2007) doi:10.1103/PhysRevD.76.034017 [arXiv:0705.2025 [hep-ph]].

[24] G. Isidori, F. Mescia and C. Smith, Nucl. Phys. B 718, 319 (2005) doi:10.1016/j.nuclphysb.2005.04.008 [hep-ph/0503107].

[25] G. Buchalla and A. J. Buras, Nucl. Phys. B 412, 106 (1994) doi:10.1016/0550-3213(94)90496-0 [hep$\mathrm{ph} / 9308272]$.

[26] K. Agashe, A. E. Blechman and F. Petriello, Phys. Rev. D 74, 053011 (2006) doi:10.1103/PhysRevD.74.053011 [hep-ph/0606021].

[27] A. M. Baldini et al. [MEG Collaboration], Eur. Phys. J. C 76, no. 8, 434 (2016) doi:10.1140/epjc/s10052016-4271-x [arXiv:1605.05081 [hep-ex]].
[28] U. Bellgardt et al. [SINDRUM Collaboration], Nucl. Phys. B 299, 1 (1988). doi:10.1016/05503213(88)90462-2

[29] J. Kaulard et al. [SINDRUM II Collaboration], Phys. Lett. B 422, 334 (1998). doi:10.1016/S03702693(97)01423-8

[30] A. L. Fitzpatrick, G. Perez and L. Randall, Phys. Rev. Lett. 100, 171604 (2008) doi:10.1103/PhysRevLett.100.171604 [arXiv:0710.1869 [hep-ph]].

[31] G. Perez and L. Randall, JHEP 0901, 077 (2009) doi:10.1088/1126-6708/2009/01/077 [arXiv:0805.4652 [hep-ph]].

[32] K. w. Choi, D. Y. Kim, I. W. Kim and T. Kobayashi, Eur. Phys. J. C 35, 267 (2004) doi:10.1140/epjc/s200401749-9 [hep-ph/0305024].

[33] D. Marti and A. Pomarol, Phys. Rev. D 64, 105025 (2001) doi:10.1103/PhysRevD.64.105025 [hep-th/0106256].

[34] E. Dudas and G. von Gersdorff, JHEP 1210, $100 \quad$ (2012) doi:10.1007/JHEP10(2012)100 [arXiv:1207.0815 [hep-th]].

[35] E. Dudas and A. M. Iyer and S. K. Vempati, To appear [arXiv:1801.xxxx ]. 